\title{
Self-Assembly Controls Reactivity with Nitric Oxide: Implications for Fluorescence Sensing
}

\author{
Carles Felip-León, César A. Angulo-Pachón, Juan F. Miravet,*(i) and Francisco Galindo*(1) \\ Departamento de Química Inorgánica y Orgánica, Universitat Jaume I, Avda. Sos Baynat s/n, 12071 Castellón, Spain \\ Supporting Information
}

ABSTRACT: Three molecules containing the fluorophore 4amino-1,8-naphthalimide (ANI) and showing different tendencies to self-assembly in aqueous environment have been prepared and fully characterized. The fluorescence emissions of two of these compounds in aqueous solutions are efficiently quenched in the presence of nitric oxide (NO) in aerated medium. Nuclear magnetic resonance and mass spectrometry techniques indicate that $\mathrm{NO} / \mathrm{O}_{2}$ induces deamination of the ANI fluorophore, resulting in nonemissive 1,8-naphtalimide derivatives. It is found that the reactivity toward $\mathrm{NO} / \mathrm{O}_{2}$ is regulated by the different aggregation modes presented by the molecules in aqueous medium. In this way, the molecules

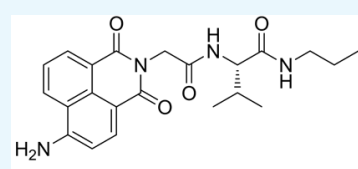

Nitric oxide sensitive

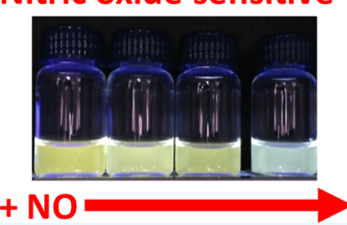

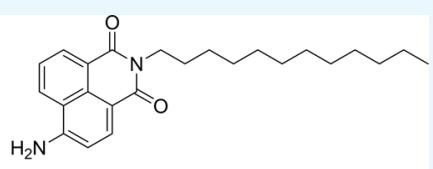

Nitric oxide insensitive

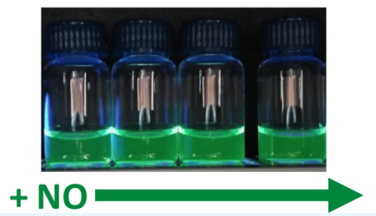
displaying fluorescence response toward $\mathrm{NO} / \mathrm{O}_{2}$ are those with weak self-association properties whereas the compound with a high hydrophobic character (self-assembling into large nanoparticles) is insensitive to this species. Ultimately, the results described here could not only set the basis for the design of fluorescent bioprobes for $\mathrm{NO} / \mathrm{O}_{2}$ based on ANI derivatives or other monoamino compounds but also could raise awareness about the importance of supramolecular interactions for the design of chemosensors.

\section{INTRODUCTION}

Nitric oxide (NO) is a molecule playing numerous roles at the biological level. ${ }^{1}$ It has been identified as a messenger molecule, ${ }^{2}$ a vasodilator, ${ }^{3}$ antibacterial agent, ${ }^{4,5}$ and also has been associated to disorders such as cancer ${ }^{6}$ or Alzheimer's disease. ${ }^{7}$ Hence, its detection by spectroscopic techniques is of great practical importance not only to develop diagnostic tools but also for the understanding of the biochemical mechanisms of action of this elusive molecule. A series of approaches have been followed to detect NO in biological samples, such as the use of electron spin resonance, ${ }^{8}$ electrochemistry, ${ }^{9}$ or fluorescence spectroscopy. ${ }^{10}$ The latter technique has unique advantages, such as high sensitivity and spatiotemporal resolution. ${ }^{11}$ A great number of fluorescent probes have been described in the literature so far, ${ }^{12-29}$ and a series of excellent reviews summarize different chemical strategies followed by a plethora of researchers in this field. ${ }^{10,30-33}$ The most frequently used strategy for NO detection is the utilization of probes containing the ortho-phenylenediamino structure. The reaction of such units with nitric oxide, in aerated medium, is well-known, yielding triazole aromatic derivatives whose optical properties are distinct from those of the original diamine molecule. However, this approach, although extremely useful for elucidating the mechanisms of action of NO, suffers from some drawbacks. One of such issues is the complexity of the synthetic routes followed to make the sensor, often leading to only a few milligrams of the probe. Another problem is selectivity, since it has been reported, in some cases, that dicarbonyl metabolites, such as dehydroascorbic acid (DHA) and methylglyoxal (MGO, pyruvaldehyde), lead to emissive products that can be wrongly interpreted as positives for NO. ${ }^{34-38}$ An alternative approach consists in the use of monoamino aromatic compounds, taking advantage of the well-known reaction of amino groups with NO in oxygenated media to afford diazonium salts, which, under certain circumstances, leads to loss of $\mathrm{N}_{2} \cdot{ }^{39-41}$ This deamination strategy has been already explored in a limited number of cases in the field of sensing. For instance, Wang and co-workers used a fluorescein derivative bearing a single amino group which, upon reaction with $\mathrm{NO}$ in aerated aqueous medium, led to a deaminated product displaying strong fluorescence. ${ }^{42}$ In another example, Guo and co-workers have reported BODIPY dyes bearing monoamino functionalities that react with $\mathrm{NO}$ in aerated medium with notable velocity. ${ }^{43}$ Recently, an acustogenic probe for NO has been designed using the reactivity of monoamino aromatics. ${ }^{44}$ We have reported a series of pyrylium dyes for NO bioimaging following analogous principles. ${ }^{18}$ The simplicity of introducing a single amino group in the sensing system is advantageous from the synthetic point of view, and it has allowed to produce nonconventional NO sensing structures. For instance, a metal-organic framework was developed by the group of Gosh using this type of

Received: August 2, 2018

Accepted: October 31, 2018

Published: November 15, 2018 
Scheme 1. Studied Molecules and Reactivity Toward Aerated NO or Acidic Nitrite<smiles>[R]N1C(=O)c2cccc3c(N)ccc(c23)C1=O</smiles><smiles>CC(C)[C@H](C)C(=O)O[N+](=O)[O-]</smiles><smiles>[R]N1C(=O)c2cccc3cccc(c23)C1=O</smiles>

1

2

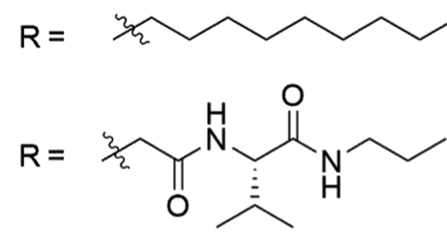

approach. $^{26}$ The same strategy is also valid for the detection of nitrite $\left(\mathrm{NO}_{2}{ }^{-}\right)$anion since this species, in acidic medium, is able to perform the conversion of the amino group into the diazonium moiety via the intermediate nitrous anhydride $\left(\mathrm{N}_{2} \mathrm{O}_{3}\right)$. Thus, systems for the analysis of $\mathrm{NO}_{2}{ }^{-}$include functionalized gold nanoparticles with organic ligands bearing aniline units ${ }^{45}$ or upconversion nanoparticles attached to a dye having a primary amino group in its structure. ${ }^{46}$ Considering the promising perspectives of this approach for the development of nitric oxide and nitrite sensors, it is likely that in the future, a number of probes based on compounds with a single $-\mathrm{NH}_{2}$ group will be developed.

One of the most popular fluorophores used so far in the broad area of fluorescence chemical sensing and bioimaging is 4-amino-1,8-naphthalimide (ANI). It has been used to develop sensors and a number of materials of varied functions. ${ }^{47,48} \mathrm{We}$ envisaged that the amino group present in this fluorophore could be removed by reaction with $\mathrm{NO}$ in aerated medium (and hence also by acidic nitrite), and as a consequence, this moiety could be used for the development of optical probes for NO and nitrite (see Scheme 1). In this paper, we explore the feasibility of that idea by testing three derivatives containing an ANI unit. Recently, we reported that compound 3 consisting of an ANI fluorophore linked to the dipeptide GlyVal formed self-assembled fibrillary networks and their optical properties were described in acetonitrile. ${ }^{49}$ Here, we analyze the reactivity of this compound and the analogs $\mathbf{1}$ and $\mathbf{2}$ in aerated aqueous media toward NO. Two points are specifically addressed here: on one hand, the reactivity of the ANI moiety and on the other hand, how it is affected by the tendency of aggregation of the reacting molecules. Overall, this study is aimed to provide a conceptual background for future optimized fluorescent sensors based on this fluorophore.

\section{RESULTS AND DISCUSSION}

Aggregation Behavior. Compound 1 showed no aggregation in the experimental conditions used $\left(\mathrm{H}_{2} \mathrm{O}\right.$ / dimethyl sulfoxide (DMSO) 9:1, $10 \mu \mathrm{M}$ ), forming transparent solutions even at concentrations in the millimolar range. On the other hand, compounds 2 and 3 form aggregates under these conditions, which could be detected by dynamic light scattering (DLS) (Figure 1), transmission electron microscopy (TEM), and scanning electron microscopy (SEM). DLS (number-averaged) analysis revealed monomodal distributions corresponding to nanoparticles with a diameter of $131 \pm 5 \mathrm{~nm}$ in the case of compound 3 and $422 \pm 14 \mathrm{~nm}$ in the case of

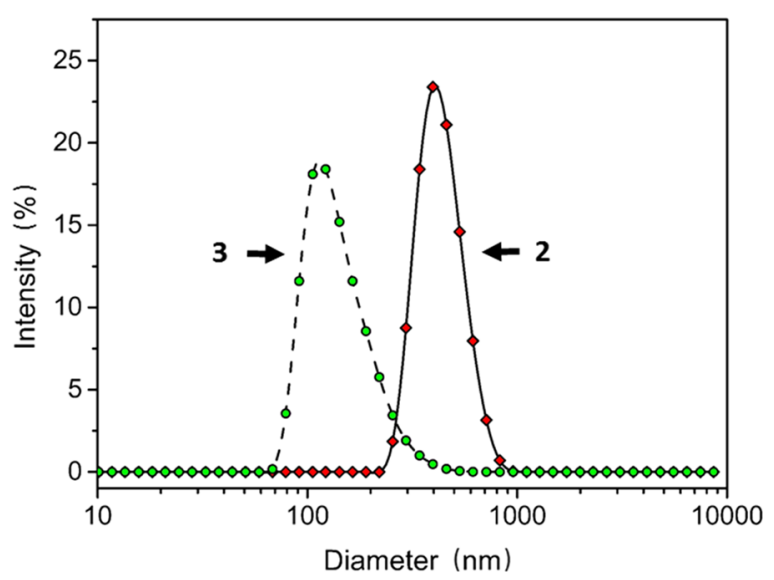

Figure 1. DLS analysis (number-averaged diameter, $D_{\mathrm{n}}$ ) of compounds 2 and 3 showing nanoparticles of $D_{n}=422 \pm 14$ and $131 \pm 5 \mathrm{~nm}$, respectively.

compound 2. TEM and SEM images of nanoparticles (Figure $2 \mathrm{a}, \mathrm{c})$ are in agreement with these observations, showing spherical objects whose size is in agreement with that observed

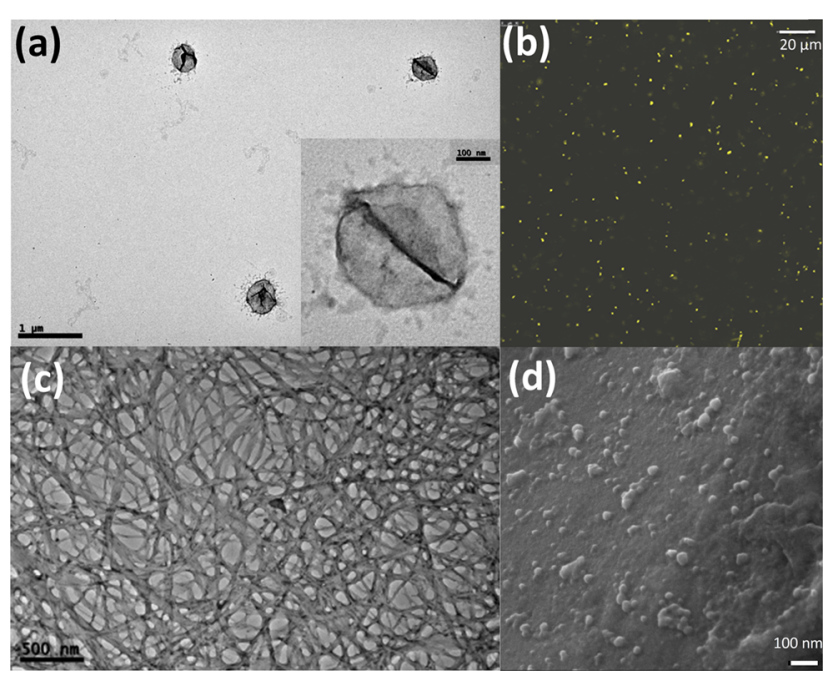

Figure 2. (a) TEM images of suspensions of 2 showing spherical nanoparticles of around $400 \mathrm{~nm}$; inset: detail of one nanoparticle; (b) CLSM showing fluorescent aggregates of 2; (c) TEM Images of gels of 3 showing fibrillar structures (high concentration); (d) SEM images of nanoparticles of 3 (low concentration). 
by DLS. In the case of compound 2 , aggregates could be visualized by confocal laser scanning microscopy (CLSM), revealing a uniform distribution of rather monodisperse fluorescent objects (Figure $2 \mathrm{~b}$ ). The formation of micro and nanoparticles in aqueous media by an analog of compound 3 has been recently described by us. ${ }^{50}$ On moving from micromolar to millimolar concentrations, compound 3 forms molecular gels composed of self-assembled fibrillar networks (Figure 2c) whereas compound 2 affords amorphous solids.

The analysis of the fluorescence of stabilized suspensions of 2 and 3 suggests that the arrangement of ANI units in the selfassembled structures differs notably. As can be seen in Figure 3 , the fluorescence maximum of 3 occurs at $546 \mathrm{~nm}$ (yellow

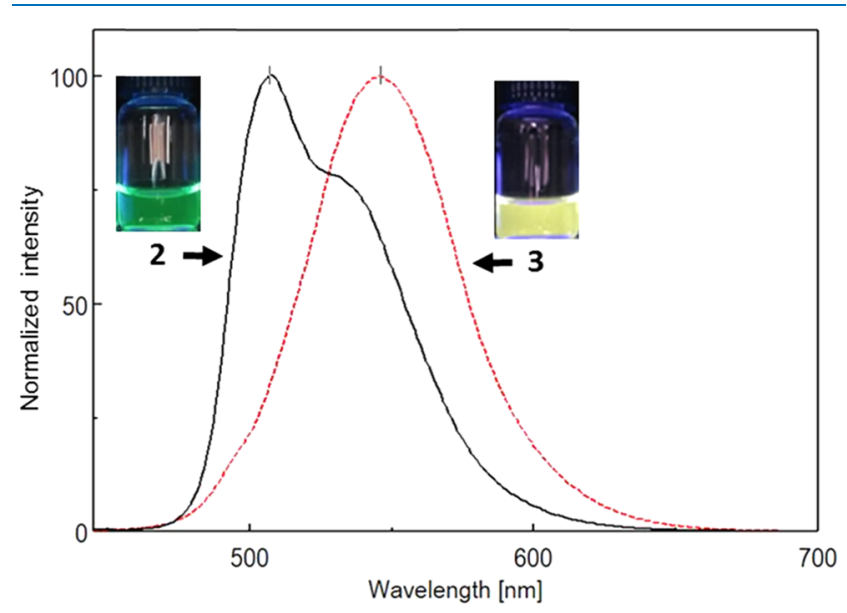

Figure 3. Normalized fluorescence spectra of suspensions of 2 and 3 at $10 \mu \mathrm{M}$ in $\mathrm{H}_{2} \mathrm{O} / \mathrm{DMSO}(9: 1) ; \lambda_{\text {exc }}=433 \mathrm{~nm}$.

color emission) whereas that of 2 experiences a strong hypsochromic shift to $507 \mathrm{~nm}$ (green color emission). These differences could be ascribed to a different solvation of the ANI units: the emitting fluorophores in 3 would be exposed to the aqueous environment (in such polar environment the emission would have a strong ICT character, typical of the ANI fluorophore); however, the environment of $\mathbf{2}$ would be clearly hydrophobic and hence the ANI moieties would be surrounded by organic residues, hidden away from the aqueous medium. Compound 1, bearing a carboxylic acid functional group and fully soluble, emits yellow fluorescence, same as 3, with a maximum at $546 \mathrm{~nm}$.

Reactivity Toward $\mathrm{NO}$ and $\mathrm{NO}_{2}{ }^{-}$. The reactivity of $1-3$ toward $\mathrm{NO}$ and $\mathrm{NO}_{2}^{-}$was assayed for $10 \mu \mathrm{M}$ samples in $\mathrm{H}_{2} \mathrm{O} /$ DMSO (9:1). First, a preliminary test was conducted with 3 in the presence of NO excess (bubbled), revealing a complete quenching of the fluorescence emission of this compound at $546 \mathrm{~nm}$. An analogous assay was conducted to test the reactivity toward acidic nitrite. Hence, when a solution of nanoparticles of 3 was exposed to $0.5 \mathrm{mM}$ nitrite in $2 \%$ acetic acid, a rapid loss of emission took place, along with a change in the color of the suspension. To rationalize these results, an analysis of the reaction with $\mathrm{NO}$ using nuclear magnetic resonance (NMR) was conducted. Comparison of the ${ }^{1} \mathrm{H}$ NMR spectrum of the reaction product with the spectrum of a model compound without the amino group (see Figure 4), prepared as reported elsewhere, ${ }^{50}$ confirmed that deamination was the main reaction taking place.

Deamination of $\mathbf{3}$ was unequivocally confirmed by mass spectrometry, as can be seen in Figure 5. Therefore, it can be concluded that the reactivity toward aerated NO of the ANI fluorophore proceeds via deamination. As a matter of fact, the deamination of anilines is a well-known reaction described frequently for synthetic purposes. ${ }^{39-41}$ It is important to remark that loss of $\mathrm{NH}_{2}$ is the sole reaction taking place in our specific conditions, although other potential reactions could occur, such as hydroxylation (already well documented for other monoamino aromatics). ${ }^{51,52}$ Nevertheless, the important idea to remark here is that reactivity of a single amino group can lead to important optical changes and hence can be used for bioanalytical purposes, as other sensors described in the

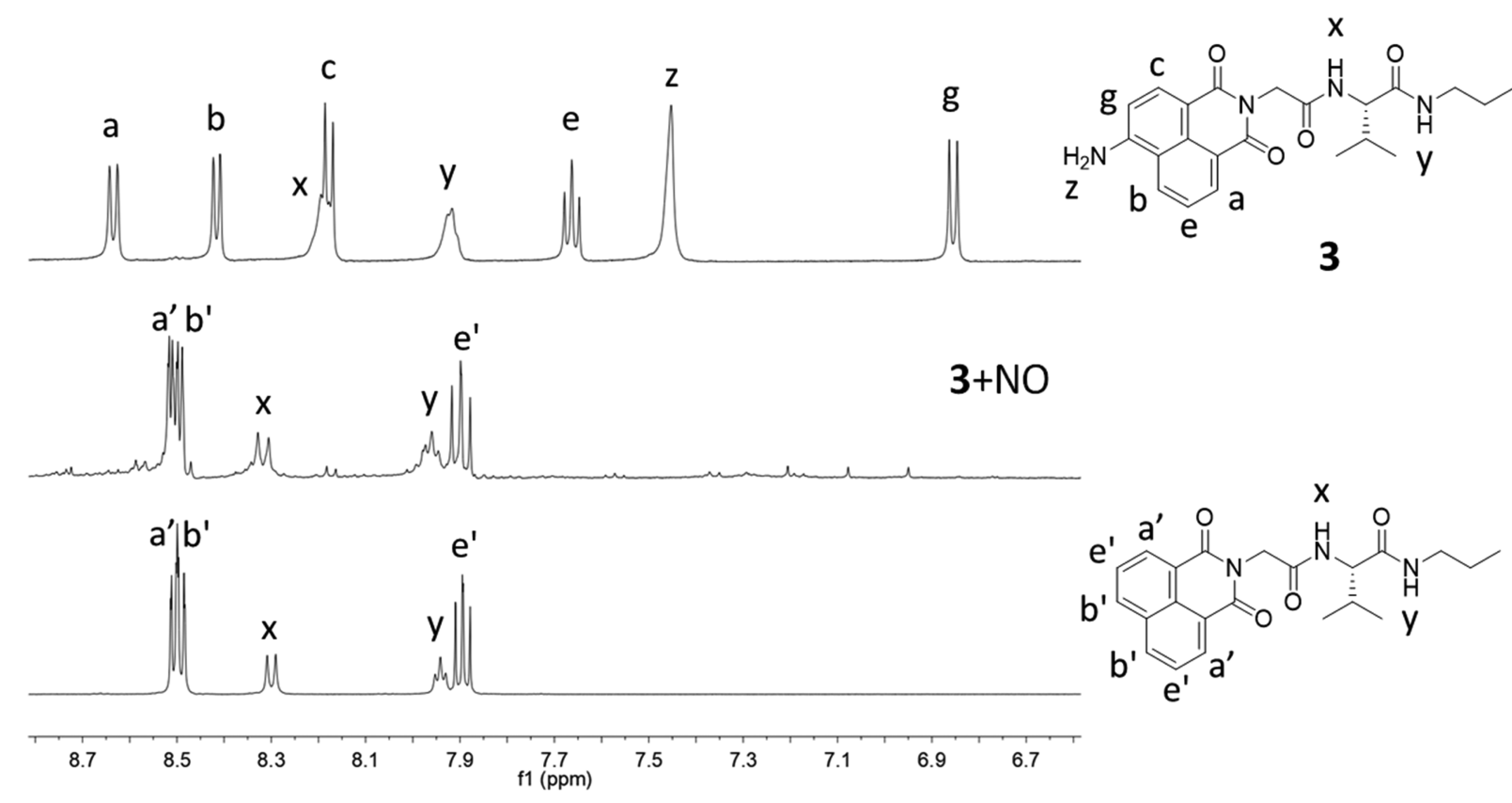

Figure 4. From top to bottom: ${ }^{1} \mathrm{H}$ NMR spectra of 3 before reaction, after reaction with NO (g) excess, and of a model compound without the amino group (synthesized as described in ref 50). 


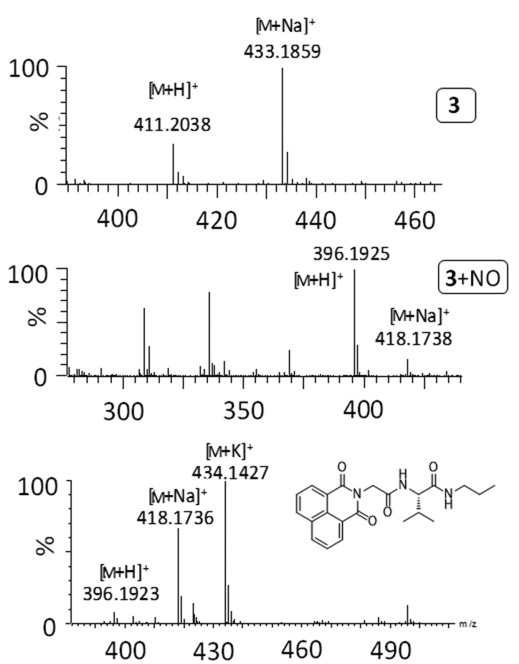

Figure 5. From top to bottom: high-resolution mass spectra of 3 before reaction, after reaction with $\mathrm{NO}(\mathrm{g})$ excess, and model compound without the amino group (synthesized as described in ref $50)$.

Introduction section. However, other elements of the molecular architecture, not only the emitting fluorophore, should be also considered, as will be shown in the following discussion, since they play a key role in the feasibility of this strategy.

Next, the fluorescence responses of 1-3 toward controlled concentrations of NO were compared. To this end, the NO donor 1,1-diethyl-2-hydroxy-2-nitroso-hydrazine sodium salt (DEA NONOate) was used. ${ }^{53}$ Incremental amounts of DEA NONOate were added to $10 \mu \mathrm{M}$ samples of $1-3$, and the response was monitored by fluorescence spectroscopy. The intensity of emission at the maximum was plotted against the concentration of released NO. It is important to note that samples are air-equilibrated otherwise the reaction does not proceed. The formation of the nitrosating species nitrous anhydride is a requisite for the reaction to occur, since the loss of nitrogen proceeds via the formation of the intermediate diazonium salt, as described in the literature for other aromatic amines. $^{39-41}$

Compounds 1 and 3 react with aerated NO in a dosedependent manner, as can be seen in Figure 6. From the onset of the curves in Figure 6, an approximate limit of detection

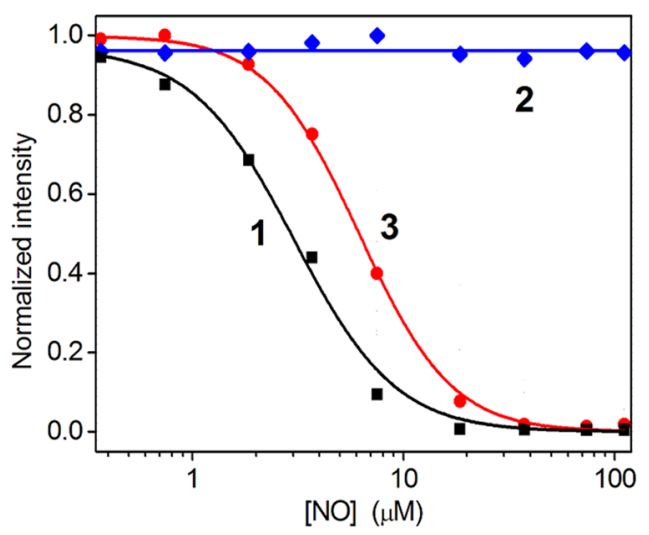

Figure 6. Normalized fluorescence intensity of $\mathbf{1 - 3}$ vs $[\mathrm{NO}]$ in $\mathrm{H}_{2} \mathrm{O} /$ DMSO (9:1), $2 \%$ AcOH. $[1-3]=10 \mu \mathrm{M} ;[\mathrm{NO}]=0.37,0.74,1.8$, $3.7,7.5,18.5,37.5,73.5$, and $111 \mu \mathrm{M} ; \lambda_{\text {exc }}=420 \mathrm{~nm}$.
(LOD) of $0.5 \mu \mathrm{M}$ for 1 and $2 \mu \mathrm{M}$ for 3 can be estimated. The assay was repeated with 3 in the presence of less DMSO (only $1 \%$ ), resulting also in the same sensitivity (see Supporting Information, Figure S7). Although stabilization times for the above experiments were set at $8 \mathrm{~h}$ to assure completion of the reactions, it must be mentioned that these processes are actually much faster. As it can be seen in the Supporting Information, completion of the deamination reaction can take place in a much shorter time. Moreover, the reactions were repeated in the presence of DHA $(10 \mu \mathrm{M})$ and MGO $(10 \mu \mathrm{M})$ as potential interfering species and, as can be seen in the Supporting Information, the effect on the reactivity was negligible.

The different behaviors shown by 1 and 3 could be attributable to the fact that $\mathbf{1}$ remains completely soluble in the aqueous medium due to the presence of a carboxylic acid in its structure whereas 3 tends to form small nanoparticles (see DLS, TEM, and SEM results presented above). But the most striking behavior is that of compound 2 , which is completely insensitive to $\mathrm{NO}$ in the assayed concentration range. In Figure 7 , a representative set of spectra can be seen for compounds 2

(a)

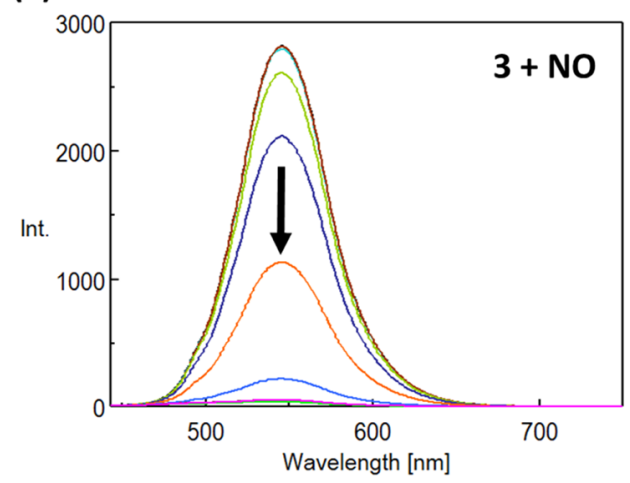

(b)

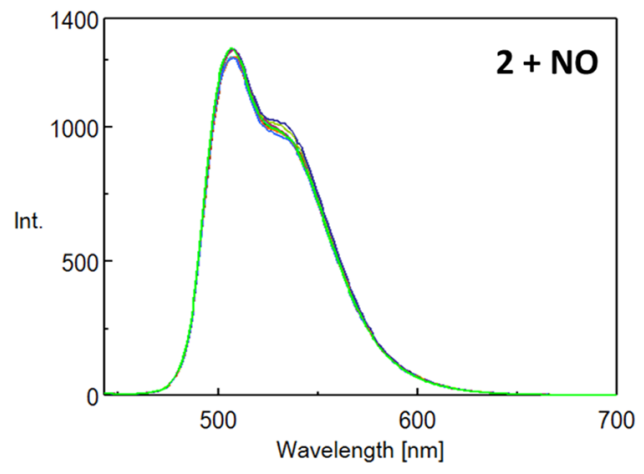

Figure 7. Representative variation of fluorescence spectra of compounds 3 (a) and 2 (b) with increasing concentrations of $\mathrm{NO}$ $(0,0.37,0.74,1.8,3.7,7.5,18.5,37.5,73.5$, and $111 \mu \mathrm{M})$ in $\mathrm{H}_{2} \mathrm{O} /$ DMSO (9:1) and $2 \%$ AcOH. $\lambda_{\text {exc }}=420 \mathrm{~nm}$.

and 3. To explain the difference, the distinct emission shown by 2 (green fluorescence) as compared to that of $\mathbf{1}$ and 3 (yellow fluorescence) must be recalled. Previously, it has been suggested that the different emission must be related to the distinct environment of the ANI fluorophores, solvated by water molecules in the case of $\mathbf{1}$ and 3 or buried in a hydrophobic aggregate in the case of $\mathbf{2}$. The lack of reactivity of 2 toward $\mathrm{NO} / \mathrm{O}_{2}$ supports this hypothesis. Besides, it 
should be noticed that compound $\mathbf{2}$ is much more hydrophobic $(C \log P=7.7)$ than $3(C \log P=2.5)$ and therefore rather strong solvophobic interactions are most likely present in the aggregates of $\mathbf{2}$.

An additional experiment was conducted to get a confirmation of the solvophobic environment of the ANI fluorophores in the stabilized suspensions of 2 . It was hypothesized that, prior to the formation of the observed large nanoparticles (see Figure 1), the individual molecules solvated in the aqueous medium could be detected, if measurements were taken at the beginning of the self-assembly process. Thus, a solution of $\mathbf{2}$ in DMSO was injected into water (reaching a ratio $\mathrm{H}_{2} \mathrm{O} / \mathrm{DMSO}$ of 9:1) and the emission spectrum of the suspension was monitored from the moment of the injection. As can be seen in Figure 8a, the emission at $t=$ 0 min displays a maximum at $551 \mathrm{~nm}$, thus revealing the fluorophore in an aqueous environment (like $\mathbf{1}$ and 3) but this fluorescence changes gradually with time until it is transformed into the green emission peaking at $507 \mathrm{~nm}$ (with a certain contribution of the long wavelength emission, as can be noticed by the shoulder in the spectrum). The kinetics of this aggregation is shown in Figure $8 \mathrm{~b}$. Aliquots of this suspension were simultaneously analyzed by DLS (Figure 8c) and a drastic increase in the diameter of the nanoparticles was found, in close parallelism with the changes in the emission profiles. This fact confirms the transformation from free molecules to aggregates where the ANI fluorophores are buried inside a hydrophobic environment, not accessible to $\mathrm{NO} / \mathrm{O}_{2}$.

\section{CONCLUSIONS}

The reactivity of three ANI derivatives, 1-3, toward dissolved nitric oxide in aerated medium has been studied. Compound 1 is fully soluble in aqueous medium, but $\mathbf{2}$ and 3 tend to aggregate. DLS, electron microscopy (SEM, TEM), and confocal microscopy studies reveal that compounds $\mathbf{2}$ and $\mathbf{3}$ self-associate to form nanoparticles with diameters of ca. 420 and $130 \mathrm{~nm}$, respectively. It has been found that the ANI fluorophore experiences deamination reaction in the presence of $\mathrm{NO} / \mathrm{O}_{2}$, with the concomitant fluorescence quenching process. This phenomenon is found to be dependent on the particular architecture of the studied molecules and their supramolecular organization. Nonaggregating molecule $\mathbf{1}$ is the species displaying the highest sensitivity (approx. LOD 0.5 $\mu \mathrm{M}$ ) followed by 3 (approx. LOD $2 \mu \mathrm{M}$ ). Remarkably, in the case of 2, aggregation into large nanoparticles where the ANI fluorophore is hidden from the aqueous environment blocks the reactivity toward $\mathrm{NO} / \mathrm{O}_{2}$.

These findings could have implications at two different levels. On the one hand, they are intended to give experimental support for the development of new indicators for NO (also for $\mathrm{NO}_{2}{ }^{-}$since they work on the basis of similar mechanisms), based on monoamines, instead of the frequently used orthophenylenediamino approach. This line is especially important if taking into account that this type of well-known probes can suffer interferences from DHA and MGO (which is not the case for the monoamine sensors under the studied conditions). The sensitivity of the molecules studied here lies within the micromolar range, which could be improved in future work by activation of the intrinsically electron-deficient 1,8-naphthalimide ring. Although the turn-off behavior is not the ideal one for a fluorescent probe, it could be used in combination with another fluorophore, utilizing the Förster resonance energy transfer mechanisms for ratiometric measurements (work in (a)

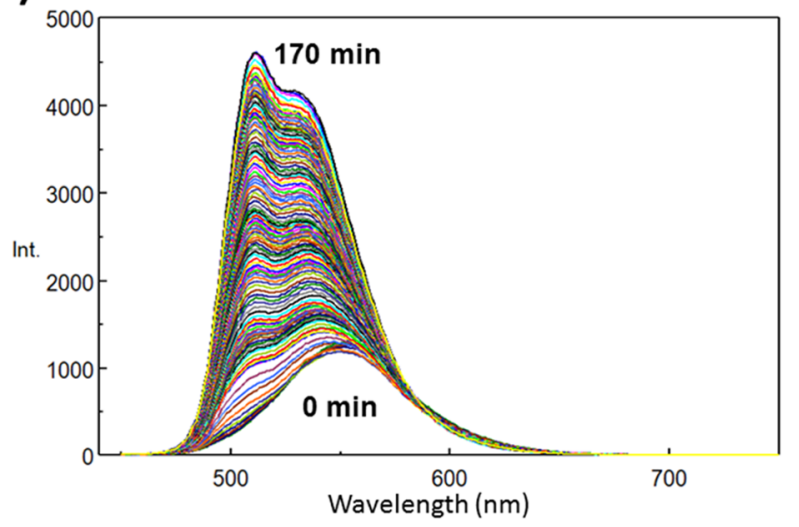

(b)
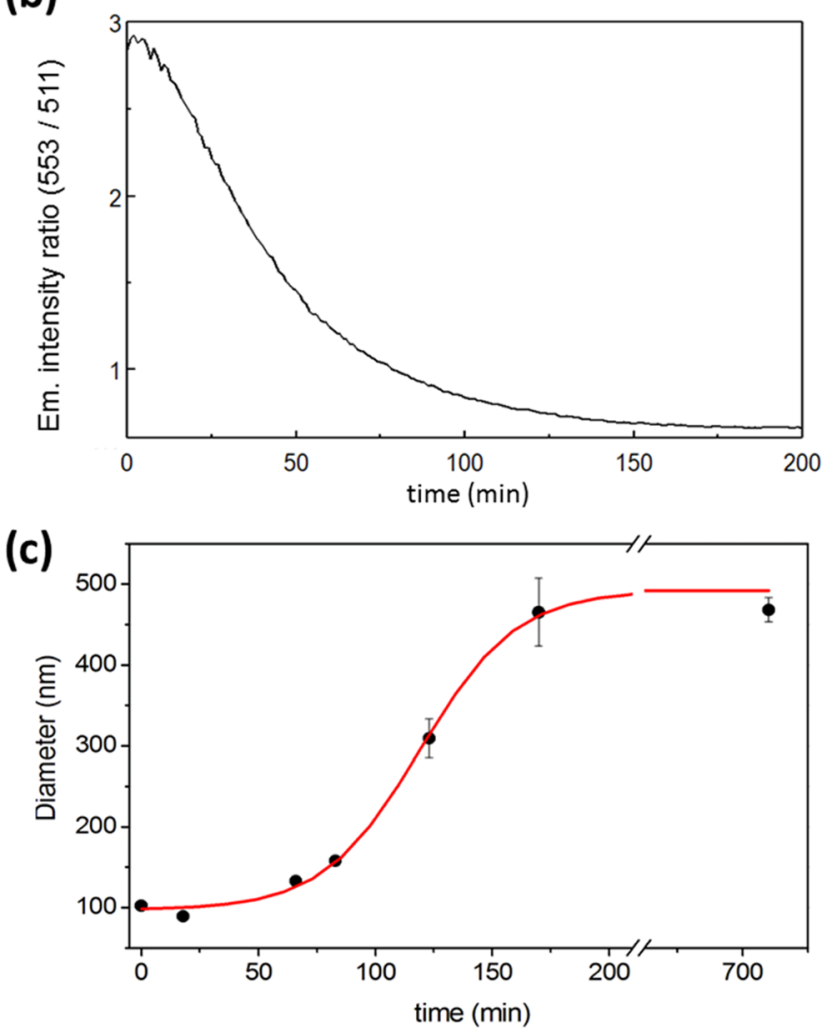

Figure 8. (a) Spontaneous evolution (self-association) of the fluorescence of a sample of $\mathbf{2}$ in aqueous environment (compound 2 is initially in DMSO and injected into water, as detailed in the main text); (b) monitorization of the fluorescence intensity occurring after injection of 2 into water; and (c) parallel monitorization of the formation of nanoparticles of $\mathbf{2}$ by means of DLS.

progress), same as for other sensors reported in the literature. ${ }^{2,49,54,55}$ On the other hand, this study aims to raise awareness about the fact that aggregation of any sensing molecule can have a profound impact on its sensitivity and hence warns about the possibility of missing data in bioimaging assays due to the self-assembly of the probe.

\section{EXPERIMENTAL SECTION}

Synthesis. General Procedure for Coupling Amino Acid and Amine. A solution of $\mathrm{Et}_{3} \mathrm{~N}(2.30 \mathrm{~mL}, 16.3 \mathrm{mmol}, 1$ eqiv $)$ in tetrahydrofuran (THF) $(40 \mathrm{~mL})$ was added dropwise to a solution of commercially available carbobenzyloxy-L-amino acid $(16.22 \mathrm{mmol})$ in THF $(60 \mathrm{~mL})$ under $\mathrm{N}_{2}$ flow on an ice 
bath at $0{ }^{\circ} \mathrm{C}$. To this mixture, an ice bath-cooled solution of ethyl chloroformate $(1.70 \mathrm{~mL}, 17.7 \mathrm{mmol}, 1.1$ eqiv) in THF $(50 \mathrm{~mL})$ was added dropwise. The reaction was left at $0{ }^{\circ} \mathrm{C}$ for $30 \mathrm{~min}$. Finally, a solution of amine (19.8 mmol, 1.2 eqiv) in THF $(50 \mathrm{~mL})$ was added dropwise for $15 \mathrm{~min}$ at $0{ }^{\circ} \mathrm{C}$. The reaction was left for $12 \mathrm{~h}$ at room temperature. Thereafter, the solvent was removed under reduced pressure and the residue was poured into $0.1 \mathrm{M} \mathrm{HCl}$ and the mixture was sonicated for $5 \mathrm{~min}$. It was filtered under vacuum, and the residue was washed with $0.1 \mathrm{M} \mathrm{KOH}$ and $\mathrm{H}_{2} \mathrm{O}$ until $\mathrm{pH}=7$. The residue was dried under reduced pressure at $50{ }^{\circ} \mathrm{C}$ overnight.

General Procedure for Deprotection of Carbobenzyloxy Group. Palladium catalyst $(10 \% \mathrm{w} / \mathrm{w})$ was suspended in $\mathrm{MeOH}(250 \mathrm{~mL})$ and stirred under $\mathrm{H}_{2}$ at room temperature for $5 \mathrm{~min}$. Subsequently, a solution of carbobenzyloxy amino compound in $\mathrm{MeOH}(150 \mathrm{~mL})$ was added via a syringe, followed by stirring under $\mathrm{H}_{2}$ at room temperature for $2-4 \mathrm{~h}$. The reaction mixture was then filtered through high-performance liquid chromatography filters $(0.46 \mu \mathrm{m})$, and the solvent was removed under reduced pressure to yield the respective amine.

General Procedure for Preparing 4-Nitro-1,8-Naphthalimide Derivatives. 4-Nitro-1,8-naphthalic anhydride (498.2 $\mathrm{mg}, 1.94 \mathrm{mmol}, 1$ eqiv) was suspended in a closed opaque vial with $\mathrm{CH}_{3} \mathrm{OH}(15 \mathrm{~mL})$ and vigorous stirring at room temperature. A solution of free amine compound in $\mathrm{CH}_{3} \mathrm{OH}$ $(15 \mathrm{~mL})$ was added dropwise. The reaction mixture was heated to $75{ }^{\circ} \mathrm{C}$ for $8 \mathrm{~h}$. Solution of the suspension was observed as the temperature increased, and a voluminous solid appeared. Thereafter, the reaction mixture was left at room temperature and the brown solid was filtered through a sintered funnel and washed with $\mathrm{CH}_{3} \mathrm{OH}$ several times until a light brown solid was obtained.

General Procedure for Reduction of $\mathrm{NO}_{2}$ Group. Palladium catalyst $(10 \% \mathrm{w} / \mathrm{w})$ was suspended in $\mathrm{MeOH}(50 \mathrm{~mL})$ and stirred under $\mathrm{H}_{2}$ at room temperature for $10 \mathrm{~min}$. Subsequently, a suspension of 4-nitro-1,8-naphthalimide derivatives in $\mathrm{MeOH}(50 \mathrm{~mL})$ was added via a syringe, followed by stirring under $\mathrm{H}_{2}$ at room temperature for $12 \mathrm{~h}$. The solution changes color almost immediately from a brown suspension to a green/yellow suspension. The palladium catalyst is removed by filtering with celite. To recover the entire product, we keep washing with DMSO. The $\mathrm{CH}_{3} \mathrm{OH}$ was evaporated, and the DMSO was lyophilized to obtain a pure product.

Procedure for Ester Hydrolysis. Compound 10 (see ESI, Supporting Information) was dissolved in $\mathrm{THF} / \mathrm{H}_{2} \mathrm{O}$ mixture $(1 / 1)$ in presence of 10 eqiv of $\mathrm{LiOH}$ and heated to $60{ }^{\circ} \mathrm{C}$ for $6 \mathrm{~h}$. The reaction mixture was evaporated under vacuum; next, the aqueous mixture was acidified $(\mathrm{HCl} 1 \mathrm{M})$ to $\mathrm{pH}=1$. A red solid were precipitated, filtered, and washed with distilled water to obtain a pure product.

Materials. Commercial reagents 1,1-diethyl-2-hydroxy-2nitroso-hydrazine sodium salt (NONOate), $\mathrm{NaNO}_{2}$, and all solvents have been used as received. ${ }^{1} \mathrm{H}$ NMR spectra were recorded at 400 and $300 \mathrm{MHz} .{ }^{13} \mathrm{C}$ NMR spectra were recorded at 126 and $75 \mathrm{MHz}$ (see ESI, Supporting Information) at $30{ }^{\circ} \mathrm{C}$ with TMS as the internal standard. Mass spectra were run in QTOF electrospray (ESMS) mode.

Fluorescence and UV-Vis Spectroscopy. Solutions and suspensions were measured in a JASCO FP-8300 spectrofluorometer using $3 \mathrm{~mL}$ quartz cuvettes with $10 \mathrm{~mm}$ path length at $20{ }^{\circ} \mathrm{C}$. For UV-vis measurements, a JASCO V-630 apparatus was used.

NO Sensing. Procedure for determining the sensitivity of the synthesized products to NO was carried out by adding different amounts of concentrated stock solutions of 4-amino1,8-naphthalimide derivatives in DMSO $(1 \mathrm{mM})$ to $\mathrm{H}_{2} \mathrm{O} /$ DMSO (9:1) to achieve a final concentration of $10 \mu \mathrm{M}$ sensors in aqueous solution. Freshly prepared stock solutions of NO donor NONOate sodium in water at $\mathrm{pH} 8$ (stable at this $\mathrm{pH}$ ) were added to $3 \mathrm{~mL}$ of the previous sensor solution to have different $\mathrm{NO}$ concentrations $([\mathrm{NO}]=0,0.37,0.74,1.65,3.7$, $7.5,18.5,37.5,73.5$, and $111 \mu \mathrm{M})$. Then, $2 \%$ acetic acid was added to trigger the decomposition of the donor and release of $\mathrm{NO}$ (final $\mathrm{pH} 2.8$ ). The mixtures were left to stabilize for $8 \mathrm{~h}$, and then the reactivity observed using fluorescence spectroscopy $\left(\lambda_{\text {exc }}=420 \mathrm{~nm} ; \lambda_{\mathrm{em}}=540 \mathrm{~nm}\right)$. Controls were made to assure that the observed reactivity was due to $\mathrm{NO} / \mathrm{O}_{2}$ and not protonation of the amine groups (see the Supporting Information). Also, control assays were made in the presence of DHA and MGO as potential interfering species (see the Supporting Information).

Dynamic Light Scattering (DLS). DLS measurements were performed with Malvern Zetasizer nano ZS instrument. Three milliliters of $10 \mu \mathrm{M}$ solutions or suspensions in aqueous solutions was measured in $1 \mathrm{~cm}$ optical path quartz cuvettes at $25{ }^{\circ} \mathrm{C}$.

Scanning Electron Microscopy (SEM). Field-emission scanning electron micrographs were recorded on a JEOL $7001 \mathrm{~F}$ microscope equipped with a CCD digital camera. The corresponding dispersions were placed on top of an aluminum specimen mount stub, fast-freezed with liquid $\mathrm{N}_{2}$, and lyophilized overnight. The sample was then sputtered with Pt (Baltec SCD500) for $30 \mathrm{~s}$ and observed at $15 \mathrm{kV}$.

Transmission Electron Microscopy. TEM micrographs were taken on JEOL 2100 microscope with a thermionic gun $\mathrm{LaB}_{6} 200 \mathrm{KV}$ equipped with a GatanOrius high-resolution CCD camera. TEM samples were prepared over Carbon formvar copper grids by putting drops of suspension and incubating for $5 \mathrm{~min}$. The suspension excess was removed with filter paper and left overnight in a glass desiccator.

Confocal Laser Scanning Microscopy (CLSM). Experiments were performed on an inverted confocal microscope Leica TCS SP8. Images where obtained with an HC PL APO CS2 $63 \times / 1.40$ OIL immersion objective. Excitation of samples was performed with diode laser excitation $(405 \mathrm{~nm})$ and was observed with a photomultiplier tubes detector. Samples were straightforwardly prepared by adding $300 \mu \mathrm{L}$ of dispersions on sterilized Ibidi $\mu$-Slide 8 Well Glass Bottom: \#1.5H $(170 \pm 5$ $\mu \mathrm{m})$ Schott glass.

\section{ASSOCIATED CONTENT}

\section{S Supporting Information}

The Supporting Information is available free of charge on the ACS Publications website at DOI: 10.1021/acsomega.8b01869.

Experimental procedures for the preparation of naphthalimide derivatives, ${ }^{1} \mathrm{H}$ and ${ }^{13} \mathrm{C}$ NMR spectra and other fluorescence assays (PDF)

\section{AUTHOR INFORMATION}

\section{Corresponding Authors}

*E-mail: miravet@uji.es (J.F.M.). 
*E-mail: francisco.galindo@uji.es (F.G.).

\section{ORCID $\odot$}

Carles Felip-León: 0000-0002-2476-3243

Juan F. Miravet: 0000-0003-0946-3784

Francisco Galindo: 0000-0003-0826-6084

\section{Notes}

The authors declare no competing financial interest.

\section{ACKNOWLEDGMENTS}

Ministerio de Economía y Competitividad of Spain (grants CTQ2012-37735, BES-2013-063296, and CTQ2015-71004$\mathrm{R})$ and Universitat Jaume I (P1.1B2015-76) are acknowledged for financial support. C.F.-L. acknowledges SCIC (UJI) for technical assistance.

\section{REFERENCES}

(1) Ignarro, L. J. Nitric Oxide: Biology and Pathobiology; Academic Press, 2000

(2) Yuan, L.; Lin, W.; Zheng, K.; Zhu, S. FRET-Based SmallMolecule Fluorescent Probes: Rational Design and Bioimaging Applications. Acc. Chem. Res. 2013, 46, 1462-1473.

(3) Ignarro, L. J.; Buga, G. M.; Wood, K. S.; Byrns, R. E.; Chaudhuri, G. Endothelium-Derived Relaxing Factor Produced and Released from Artery and Vein Is Nitric Oxide. Proc. Natl. Acad. Sci. U.S.A. 1987, 84, 9265-9269.

(4) McMullin, B. B.; Chittock, D. R.; Roscoe, D. L.; Garcha, H.; Wang, L.; Miller, C. C. The Antimicrobial Effect of Nitric Oxide on the Bacteria That Cause Nosocomial Pneumonia in Mechanically Ventilated Patients in the Intensive Care Unit. Respir. Care 2005, 50, $1451-1456$

(5) Schairer, D. O.; Chouake, J. S.; Nosanchuk, J. D.; Friedman, A. J. The Potential of Nitric Oxide Releasing Therapies as Antimicrobial Agents. Virulence 2012, 3, 271-279.

(6) Xu, W.; Liu, L. Z.; Loizidou, M.; Ahmed, M.; Charles, I. G. The Role of Nitric Oxide in Cancer. Cell Res. 2002, 12, 311-320.

(7) de la Torre, J. C.; Stefano, G. B. Evidence That Alzheimer's Disease Is a Microvascular Disorder: The Role of Constitutive Nitric Oxide. Brain Res. Rev. 2000, 34, 119-136.

(8) Hogg, N. Detection of Nitric Oxide by Electron Paramagnetic Resonance Spectroscopy. Free Radical Biol. Med. 2010, 49, 122-129.

(9) Privett, B. J.; Shin, J. H.; Schoenfisch, M. H. Electrochemical Nitric Oxide Sensors for Physiological Measurements. Chem. Soc. Rev. 2010, 39, 1925-1935.

(10) Nagano, T.; Yoshimura, T. Bioimaging of Nitric Oxide. Chem. Rev. 2002, 102, 1235-1270.

(11) Lakowicz, J. R. Principles of Fluorescence Spectroscopy; Springer: Boston, MA, 2006.

(12) Kojima, H.; Urano, Y.; Kikuchi, K.; Higuchi, T.; Hirata, Y.; Nagano, T. Fluorescent Indicators for Imaging Nitric Oxide Production. Angew. Chem., Int. Ed. 1999, 38, 3209-3212.

(13) Kojima, H.; Hirotani, M.; Nakatsubo, N.; Kikuchi, K.; Urano, Y.; Higuchi, T.; Hirata, Y.; Nagano, T. Bioimaging of Nitric Oxide with Fluorescent Indicators Based on the Rhodamine Chromophore. Anal. Chem. 2001, 73, 1967-1973.

(14) Sasaki, E.; Kojima, H.; Nishimatsu, H.; Urano, Y.; Kikuchi, K.; Hirata, Y.; Nagano, T. Highly Sensitive Near-Infrared Fluorescent Probes for Nitric Oxide and Their Application to Isolated Organs. J. Am. Chem. Soc. 2005, 127, 3684-3685.

(15) Galindo, F.; Kabir, N.; Gavrilovic, J.; Russell, D. A. Spectroscopic Studies of 1,2-Diaminoanthraquinone (DAQ) as a Fluorescent Probe for the Imaging of Nitric Oxide in Living Cells. Photochem. Photobiol. Sci. 2008, 7, 126-130.

(16) Yuan, L.; Lin, W.; Xie, Y.; Chen, B.; Zhu, S. Single Fluorescent Probe Responds to $\mathrm{H}_{2} \mathrm{O}_{2}, \mathrm{NO}$, and $\mathrm{H}_{2} \mathrm{O}_{2} / \mathrm{NO}$ with Three Different Sets of Fluorescence Signals. J. Am. Chem. Soc. 2012, 134, 13051315.
(17) Marín, M. J.; Thomas, P.; Fabregat, V.; Luis, S. V.; Russell, D. A.; Galindo, F. Fluorescence of 1,2-Diaminoanthraquinone and Its Nitric Oxide Reaction Product within Macrophage Cells. ChemBioChem 2011, 12, 2471-2477.

(18) Beltrán, A.; Isabel Burguete, M.; Abánades, D. R.; Pérez-Sala, D.; Luis, S. V.; Galindo, F. Turn-on Fluorescent Probes for Nitric Oxide Sensing Based on the Ortho-Hydroxyamino Structure Showing No Interference with Dehydroascorbic Acid. Chem. Commun. 2014, $50,3579-3581$.

(19) Yu, H.; Xiao, Y.; Jin, L. A Lysosome-Targetable and TwoPhoton Fluorescent Probe for Monitoring Endogenous and Exogenous Nitric Oxide in Living Cells. J. Am. Chem. Soc. 2012, 134, 17486-17489.

(20) Lv, X.; Wang, Y.; Zhang, S.; Liu, Y.; Zhang, J.; Guo, W. A Specific Fluorescent Probe for NO Based on a New NO-Binding Group. Chem. Commun. 2014, 50, 7499-7502.

(21) Yu, H.; Jin, L.; Dai, Y.; Li, H.; Xiao, Y. From a BODIPYrhodamine Scaffold to a Ratiometric Fluorescent Probe for Nitric Oxide. New J. Chem. 2013, 37, 1688-1691.

(22) Sazinsky, M. H.; Lippard, S. J. Correlating Structure with Function in Bacterial Multicomponent Monooxygenases and Related Diiron Proteins. Acc. Chem. Res. 2006, 39, 558-566.

(23) Lim, M. H.; Wong, B. A.; Pitcock, W. H.; Mokshagundam, D.; Baik, M.-H.; Lippard, S. J. Direct Nitric Oxide Detection in Aqueous Solution by Copper(II) Fluorescein Complexes. J. Am. Chem. Soc. 2006, 128, 14364-14373.

(24) Pluth, M. D.; Chan, M. R.; McQuade, L. E.; Lippard, S. J. Seminaphthofluorescein-Based Fluorescent Probes for Imaging Nitric Oxide in Live Cells. Inorg. Chem. 2011, 50, 9385-9392.

(25) Mondal, B.; Kumar, P.; Ghosh, P.; Kalita, A. FluorescenceBased Detection of Nitric Oxide in Aqueous and Methanol Media Using a Copper(ii) Complex. Chem. Commun. 2011, 47, 2964-2966.

(26) Desai, A. V.; Samanta, P.; Manna, B.; Ghosh, S. K. Aqueous Phase Nitric Oxide Detection by an Amine-Decorated Metal-organic Framework. Chem. Commun. 2015, 51, 6111-6114.

(27) Apfel, U.-P.; Buccella, D.; Wilson, J. J.; Lippard, S. J. Detection of Nitric Oxide and Nitroxyl with Benzoresorufin-Based Fluorescent Sensors. Inorg. Chem. 2013, 52, 3285-3294.

(28) Yang, Y.; Seidlits, S. K.; Adams, M. M.; Lynch, V. M.; Schmidt, C. E.; Anslyn, E. V.; Shear, J. B. A Highly Selective Low-Background Fluorescent Imaging Agent for Nitric Oxide. J. Am. Chem. Soc. 2010, 132, 13114-13116.

(29) Wang, M.; Xu, Z.; Wang, X.; Cui, J. A Fluorescent and Colorimetric Chemosensor for Nitric Oxide Based on 1,8Naphthalimide. Dyes Pigm. 2013, 96, 333-337.

(30) Ye, X.; Rubakhin, S. S.; Sweedler, J. V. Detection of Nitric Oxide in Single Cells. Analyst 2008, 133, 423-433.

(31) McQuade, L. E.; Lippard, S. J. Fluorescent Probes to Investigate Nitric Oxide and Other Reactive Nitrogen Species in Biology (Truncated Form: Fluorescent Probes of Reactive Nitrogen Species). Curr. Opin. Chem. Biol. 2010, 14, 43-49.

(32) Li, H.; Wan, A. Fluorescent Probes for Real-Time Measurement of Nitric Oxide in Living Cells. Analyst 2015, 140, 7129-7141.

(33) Chen, X.; Wang, F.; Hyun, J. Y.; Wei, T.; Qiang, J.; Ren, X.; Shin, I.; Yoon, J. Recent Progress in the Development of Fluorescent, Luminescent and Colorimetric Probes for Detection of Reactive Oxygen and Nitrogen Species. Chem. Soc. Rev. 2016, 45, 2976-3016.

(34) Zhang, X.; Kim, W.-S.; Hatcher, N.; Potgieter, K.; Moroz, L. L.; Gillette, R.; Sweedler, J. V. Interfering with Nitric Oxide Measurements. 4,5-Diaminofluorescein Reacts with Dehydroascorbic Acid and Ascorbic Acid. J. Biol. Chem. 2002, 277, 48472-48478.

(35) Ye, X.; Kim, W.-S.; Rubakhin, S. S.; Sweedler, J. V. Measurement of Nitric Oxide by 4,5-Diaminofluorescein without Interferences. Analyst 2004, 129, 1200.

(36) Kim, W.-S.; Ye, X.; Rubakhin, S. S.; Sweedler, J. V. Measuring Nitric Oxide in Single Neurons by Capillary Electrophoresis with Laser-Induced Fluorescence: Use of Ascorbate Oxidase in Diaminofluorescein Measurements. Anal. Chem. 2006, 78, 1859-1865. 
(37) Shaheen, F.; Shmygol, A.; Rabbani, N.; Thornalley, P. J. A Fluorogenic Assay for Methylglyoxal. Biochem. Soc. Trans. 2014, 42, $548-555$.

(38) Wang, T.; Douglass, E. F., Jr.; Fitzgerald, K. J.; Spiegel, D. A. A. "Turn-On" Fluorescent Sensor for Methylglyoxal. J. Am. Chem. Soc. 2013, 135, 12429-12433.

(39) Burglova, K.; Okorochenkov, S.; Hlavac, J. Efficient Route to Deuterated Aromatics by the Deamination of Anilines. Org. Lett. 2016, 18, 3342-3345.

(40) Itoh, T.; Matsuya, Y.; Nagata, K.; Ohsawa, A. Reductive Deamination of Aromatic Amines with Nitric Oxide (NO). Tetrahedron Lett. 1996, 37, 4165-4168.

(41) Nagano, T.; Takizawa, H.; Hirobe, M. Reactions of Nitric Oxide with Amines in the Presence of Dioxygen. Tetrahedron Lett. 1995, 36, 8239-8242.

(42) Shiue, T.-W.; Chen, Y.-H.; Wu, C.-M.; Singh, G.; Chen, H.-Y.; Hung, C.-H.; Liaw, W.-F.; Wang, Y.-M. Nitric Oxide Turn-on Fluorescent Probe Based on Deamination of Aromatic Primary Monoamines. Inorg. Chem. 2012, 51, 5400-5408.

(43) Huo, Y.; Miao, J.; Li, Y.; Shi, Y.; Shi, H.; Guo, W. Aromatic Primary Monoamine-Based Fast-Response and Highly Specific Fluorescent Probes for Imaging the Biological Signaling Molecule Nitric Oxide in Living Cells and Organisms. J. Mater. Chem. B 2017, $5,2483-2490$.

(44) Reinhardt, C. J.; Zhou, E. Y.; Jorgensen, M. D.; Partipilo, G.; Chan, J. A Ratiometric Acoustogenic Probe for in Vivo Imaging of Endogenous Nitric Oxide. J. Am. Chem. Soc. 2018, 140, 1011-1018.

(45) Xiao, N.; Yu, C. Rapid-Response and Highly Sensitive Noncross-Linking Colorimetric Nitrite Sensor Using 4-Aminothiophenol Modified Gold Nanorods. Anal. Chem. 2010, 82, 36593663.

(46) Han, J.; Zhang, C.; Liu, F.; Liu, B.; Han, M.; Zou, W.; Yang, L.; Zhang, Z. Upconversion Nanoparticles for Ratiometric Fluorescence Detection of Nitrite. Analyst 2014, 139, 3032-3038.

(47) Duke, R. M.; Veale, E. B.; Pfeffer, F. M.; Kruger, P. E.; Gunnlaugsson, T. Colorimetric and Fluorescent Anion Sensors: An Overview of Recent Developments in the Use of 1,8-NaphthalimideBased Chemosensors. Chem. Soc. Rev. 2010, 39, 3936-3953.

(48) Abad, S.; Kluciar, M.; Miranda, M. A.; Pischel, U. ProtonInduced Fluorescence Switching in Novel Naphthalimide-Dansylamide Dyads. J. Org. Chem. 2005, 70, 10565-11568.

(49) Felip-León, C.; Díaz-Oltra, S.; Galindo, F.; Miravet, J. F. Chameleonic, Light Harvesting Photonic Gels Based on Orthogonal Molecular Fibrillization. Chem. Mater. 2016, 28, 7964-7972.

(50) Felip-León, C.; Cejudo-Marín, R.; Peris, M.; Galindo, F.; Miravet, J. F. Sizing Down a Supramolecular Gel into Micro- and Nanoparticles. Langmuir 2017, 33, 10322-10328.

(51) Hu, J.; Whittaker, M. R.; Quinn, J. F.; Davis, T. P. Nitric Oxide (NO) Endows Arylamine-Containing Block Copolymers with Unique Photoresponsive and Switchable LCST Properties. Macromolecules 2016, 49, 2741-2749.

(52) Adarsh, N.; Shanmugasundaram, M.; Ramaiah, D. Efficient Reaction Based Colorimetric Probe for Sensitive Detection, Quantification, and On-Site Analysis of Nitrite Ions in Natural Water Resources. Anal. Chem. 2013, 85, 10008-10012.

(53) Maragos, C. M.; Morley, D.; Wink, D. A.; Dunams, T. M.; Saavedra, J. E.; Hoffman, A.; Bove, A. A.; Isaac, L.; Hrabie, J. A.; Keefer, L. K. Complexes of $\cdot \mathrm{NO}$ with Nucleophiles as Agents for the Controlled Biological Release of Nitric Oxide. Vasorelaxant Effects. J. Med. Chem. 1991, 34, 3242-3247.

(54) Saura, A. V.; Marín, M. J.; Burguete, M. I.; Russell, D. A.; Galindo, F.; Luis, S. V. The Synthesis of New Fluorescent Bichromophoric Compounds as Ratiometric $\mathrm{pH}$ Probes for Intracellular Measurements. Org. Biomol. Chem. 2015, 13, 7736-7749.

(55) Vanessa Saura, A.; Burguete, M. I.; Galindo, F.; Luis, S. V. Novel Fluorescent Anthracene-bodipy Dyads Displaying Sensitivity to $\mathrm{pH}$ and Turn-on Behaviour towards $\mathrm{Cu}(\mathrm{Ii})$ Ions. Org. Biomol. Chem. 2017, 15, 3013-3024. 